\title{
25 Research Soure \\ Identification of genetic sequence variations associated with the pathogenesis of X-linked hyper-IgM syndrome
}

\section{Wang Wang ( $\sim 321860332 @ q q . c o m$ )}

The First Affiliated Hospital of Anhui Medical University https://orcid.org/0000-0002-6674-6839

Jun-Li Ding

The First Affiliated Hospital of Anhui Medical University

Yu-Lin Zhu

The First Affiliated Hospital of Anhui Medical University

Shao-Hu Huo

The First Affiliated Hospital of Anhui Medical University

Lu-Lu Fang

The First Affiliated Hospital of Anhui Medical University

Teng-Chuan Jin

The First Affiliated Hospital of Anhui Medical University

\section{Bing Shen}

The First Affiliated Hospital of Anhui Medical University

\section{Sheng-Gang Ding}

The First Affiliated Hospital of Anhui Medical University

\section{Research}

Keywords: X-HIGM, whole-exome sequencing, targeted gene sequencing, sanger sequencing, CD40LG

Posted Date: April 7th, 2021

DOI: https://doi.org/10.21203/rs.3.rs-370993/v1

License: (c) (1) This work is licensed under a Creative Commons Attribution 4.0 International License. Read Full License 


\section{Abstract}

Background: X-Linked Hyper-IgM Syndrome (X-HIGM) is a rare genetic primary immunodeficiency disease caused by mutations of the CD40 ligand gene (CD40LG). It is characterized by normal or elevated levels of IgM and markedly decreased serum IgG, IgA, and IgE levels. Patients with this syndrome are often prone to infections. Environmental and genetic (especially genetic mutation) factors may play an important role in etiology, development, and pathogenesis of X-HIGM.

Methods: DNA from a male child diagnosed as having X-HIGM and DNA from his healthy mother were used for whole-exome (next-generation) sequencing and targeted gene sequencing. The results were analyzed using Exome Aggregation Consortium data and the Genome Aggregation Database and were further validated using Sanger sequencing.

Results: Next-generation sequencing results indicated that the CD4OLG gene in the child had a p.R203I variant. In addition, his mother was a carrier, suggesting that the child's p.R203I homozygous mutation was inherited from his mother. The functional prediction scores from SIFT, MetaSVM, and FATHMM software indicated that this genetic variant may be harmful.

Conclusions: Single variations in many exons of the CD $40 L G$ gene can lead to X-HIGM. Although the pathogenicity of the variant identified in the present study has not been previously reported, prediction software found that it would be harmful. Thus, CD $40 L G$ may be related to this genetic disease. Despite these limitations, our findings provided insight into X-HIGM pathogenesis and suggested a potential target for therapeutic drug development.

\section{Introduction}

X-linked hyper-IgM syndrome (X-HIGM) is a rare genetic primary immunodeficiency disease caused by sequence variations in the gene encoding the cluster of differentiation 40 ligand $(C D 4 O L G)$. The clinical characteristics of this syndrome include normal or elevated levels of IgM but markedly decreased serum IgG, IgA, and IgE levels [1]. Environmental and genetic (especially genetic variant) factors may play an important role in the etiology, development, and pathogenesis of X-HIGM. Patients with this syndrome typically easily develop infections or opportunistic infections [2].

The CD40 ligand (CD40L) protein plays an essential role during membrane-membrane interactions between activated CD4-positive $T$ cells and $B$ cells and between CD4-positive $T$ and antigen-presenting cells [3]. The loss of the interaction between CD40 and its ligand results in impairments of T cell function, of B cell differentiation, and of monocyte function. Only B cell differentiation appears to be affected by defects of signaling molecules downstream of $\mathrm{CD} 40$, with the exception of defects in the nuclear factor-KB complex, which mediates signaling via multiple receptor pathways [4]. The product of the CD4OLG gene is a $39 \mathrm{kDa}$ type II membrane glycoprotein that is homologous to tumor necrosis factor $a$ and is expressed transiently on the surface of activated CD4-positive T cells and can also be expressed on CD8-positive T cells, B cells, mast cells, basophils, eosinophils, and platelets [5]. Therefore, finding new CD $40 L G$ variants would be of great significance and would enable a more in-depth assessment of the risk of X-HIGM.

Here, we report the findings of a genetic study assessing an 8-year-old boy with X-HIGM. We conducted whole-exome sequencing for the affected child and his healthy mother. We identified a novel CD $40 \mathrm{~L}$ mutation that was common to the affected child and his mother. Using Sanger sequencing, we verified the variations in candidate pathogenic genes for X-HIGM susceptibility. 


\section{Materials And Methods}

\section{Case Report}

A boy $(\varangle 1$, Fig. 1) was born via natural, normal childbirth at 39 weeks of gestation. His development also appeared normal and without any congenital malformation suggestive of specific syndromes. He would occasionally develop a fever and cough, but his parents paid little attention to these signs. When he was 8 years old, the boy presented to our hospital with "cough for 3 months and fever for 5 days". He was initially diagnosed with chronic pneumonia and bronchiectasis based on his clinical characteristics, blood test results, and imaging findings (Fig. 2). Serum level of immunoglobulin was IgM $5.84 \mathrm{~g} / \mathrm{L}(0.48-2.12 \mathrm{~g} / \mathrm{L})$, IgG $0.09 \mathrm{~g} / \mathrm{L}$ (7.60-16.60 g/L), IgA $0.01 \mathrm{~g} / \mathrm{L}$ (0.71-3.35 g/L), Total $\mathrm{lgE} 2 \mathrm{mlU} / \mathrm{ml}$ (20-200 mlU/ml), C3 $1.51 \mathrm{~g} / \mathrm{L}$ (0.87-1.41 g/L), C4 0.24 g/L (0.10-0.40 g/L). Based on sanger sequencing, the boy was later thought to have X-HIGM, and his serological examination showed significantly increased levels of IgM but significantly decreased levels of IgG and IgA. The child's serological results were in good agreement with clinical manifestations of X-HIGM.

\section{Editorial Policies and Ethical Considerations}

This study was approved by the Ethics Committee of Anhui Medical University. The peripheral blood of the proband and his mother were obtained at the Department of Paediatrics of the First Affiliated Hospital of Anhui Medical University. Each person volunteered to participate in this study and each person or the parent/guardian signed an informed consent form to be included in the present study. The work described was carried out in accordance with The Code of Ethics of the World Medical Association (Declaration of Helsinki).

\section{Whole-Exome Sequencing}

DNA was extracted from the peripheral blood of the proband $(\nabla 1)$ and his mother $(\varangle 3)$ using standard procedures and following the manufacturer's instructions (Hefei Novel Gene Technology Service Co., Ltd, Hefei, China). Highthroughput sequencing of DNA samples was performed after whole-exome capture. The Exome Aggregation Consortium (ExAC) data and the Genome Aggregation Database (gnomAD) were used to filter those obtained results. The filter criteria were the allele frequency of the variant in the East Asian population of the ExAC database (ExAC EAS) of $<0.05$ and of the gnomAD database (gnomAD EAS) of $<0.05$ and excluded synonymous variants or noncoding flanking regions. The remaining genes that were previously found to be closely related to tumor development were excluded. Sanger sequencing was used for final validation.

\section{Sanger Sequencing}

GenBank sequences of CD4OLG (accession number: NM-000074.2), Bruton tyrosine kinase (BTK; accession number: NM-021029.6) and Wiskott-Aldrich syndrome (WAS, accession number: NM-000373.3) were obtained from the National Center for Biotechnology Information reference sequence database. Sanger sequencing was used to verify candidate gene variants for X-HIGM susceptibility.

\section{Results}

We performed whole-exome sequencing using the genomic DNA extracted from the peripheral blood from the proband, who was diagnosed as having X-HIGM, and an apparently healthy member of his family (his mother) to 
identify candidate pathogenic genes. After we conducted bioinformatics analyses, we identified seven rare pivotal single-nucleotide variations (SNVs; formerly single-nucleotide polymorphisms [SNPs]). Of them, three were candidate genes for X-HIGM susceptibility: CD4OLG (OMIM: 300386), BTK (OMIM: 300300) and WAS (OMIM: 300392) (Table 1). Sanger sequencing was subsequently performed to verify the sequence variation in CD4OLG (Fig. 3,4). Using nextgeneration sequencing technology, we found that CD4OLG gene in the afflicted child had a p.R203I variant. We also discovered that an apparently healthy family member, the mother, was a carrier of this variant, suggesting that the child's p.R203I variant was inherited from his mother. We speculate that this child should be diagnosed as having XHIGM caused by the p.R203I variant of CD40LG gene.

\section{Discussion}

Hyper IgM (HIGM) syndromes are a group of primary immunodeficiency disorders that were defined by Burtin and Rosen in the early 1960s [6]. X-HIGM is a rare primary immunodeficiency disease. Recently, a large-scale study conducted in the United States showed that the incidence of X-HIGM was approximately 1 per 1 million persons [7]. Only about $20 \%$ of patients receiving this diagnosis reach the third decade of life [8]. In the treatment of X-HIGM, hematopoietic stem cell transplantation and immunoglobulin replacement therapy have been widely accepted [2]. Variations in CD40 ligand (CD40L, also known as CD154) gene was most commonly associated with X-HIGM, covering $65 \%-70 \%$ of HIGM cases $[9,10]$. Therefore, understanding the mechanisms of X-HIGM development and progression may be valuable for improving the diagnosis, treatment, and prognosis of children with this syndrome.

Mechanistically, CD40L protein can bind to tumor necrosis factor receptors and CD40 receptors, participate in antiapoptosis, inflammatory responses, leukocyte attachment, signal transduction, platelet activation, B cell proliferation, and isotype conversion. The pathogenesis of HIGM involves antibody class switching recombination dysfunction, with or without somatic hypermutation defects. B cells normally express IgM in the late developmental stages, and then produce IgG and IgA through class switching recombination and somatic hypermutation mechanisms. Any factors interfering with these processes can cause HIGM [11]. Variations and deletions in the CD4OLG gene in X-HIGM result in a defective CD40L protein, which, if expressed on the surface, fails to activate CD40 normally [5].

Due to the young age of onset and lack of immune antibodies, X-HIGM patients are easy to get bacterial infection, which including serve bacterial pneumonia, otitis media, sinusitis and so on, pulmonary inflammation is the most common. It has been reported that CD40LG variants can lead to dysfunction of macrophage activation, resulting in excessive accumulation of surfactants in the alveolar cavity and airways, causing pulmonary alveolar proteinosis, which may be the pathological basis of interstitial pneumonia. Also, deficiency of IgG subtype and category conversion memory B cell may lead to pulmonary fibrosis changes $[11,12]$. Pneumocystis carinii pneumonia is the most common opportunistic infection, the mechanism of it is not clear [13]. As an endolymphoid organ, liver plays an important role in immune balance, and autoimmune liver disease may be the manifestation of HIGM immune deficiency in the liver. Hepatic injury accounts for $50 \%$ of HIGM complications, which is the main cause of death in many cases. With the progress of the disease, it can evolve into sclerosing cholangitis and even cholangiocarcinoma [7]. Migration inhibition test and anti-mitochondrial antibody positive may be risk factors for X-HIGM and primary cholangitis [14]. Studies have shown that in patients with primary biliary cirrhosis, elevated serum IgM is often associated with low expression of CD40LG promoter DNA methylation [15]. Granulocyte deficiency may be associated with autoantibodies and CD40L mutations, which mediated by CD40 signaling pathway in inflammatory microenvironment can also lead to granulocyte deficiency [16]. Granulocyte colony stimulating factor can effectively improve neutrophil deficiency. And, autoimmune diseases can occur in X-HIGM patients, the appearance of 
autoantibodies lgM, immuno-tolerance of peripheral B cells and development defects of regulatory $\mathrm{T}$ cell may be responsible to this [4]. Tumor lesions are also common in X-HIGM patients, such as biliary tract tumors, neuroendocrine tumors and so on [17].

Early severe infection and liver disease are the leading causes of death of X-HIGM patients, which timely antiinfection treatment is necessary, it has been reported that even if Pneumocystis carinii was not found in bronchial lavage fluid, early anti-infection treatment should be taken if the it was suspected [18]. Intravenous immunoglobulin (IVIG) is important to improve complications and reduce mortality [19]. IVIG should be initiated actively in the early stage. The routine dose is $400 \sim 600 \mathrm{mg} / \mathrm{kg}$, once every 3 weeks, which can significantly correct humoral immune deficiency. The study found that $\gamma$ - interferon (IFN- $\gamma$ ) and TNF-a increased after percutaneous injection of recombinant $\mathrm{CD} 40 \mathrm{~L}$ in patients with immunodeficiency, which further improve the immune function of $\mathrm{T}$ cells and is beneficial to the defense of opportunistic infection and tumor immune surveillance [20]. But in vivo injection CD40L may lead to other cell line dysfunction while correcting the antibody function of B lymphocytes, and its safety needs further research [21]. Hematopoietic stem cell transplantation (HSCT) is the most effective treatment for X-HIGM. The selection of donor and pre-transplant treatment are very important for the transplantation and reconstruction of autoimmune function after operation. Human leukocyte antigen (HLA) matched sibling is the best donor of hematopoietic stem cells. However, there is a lack of sibling donors in clinic, and most of patients have to receive non-homologous donor transplantation [22]. Also, with the development of technology, gene therapy in X chain of severe diseases combined with immunodeficiency disease and adenosine deaminase deficiency has greatly promoted thetreatment process of X-HIGM. Hubbard successfully edited CD40LG genes in T cells by combining nuclease-induced double-strand breaks with donor carrying recombinant adeno-associated virus, which completly recover CD40L patient's function [23].

In the present study, we identified seven rare nonsynonymous SNVs and three genes with rare nonsynonymous SNVs (CD4OLG, BTK, and WAS) associated with immunocompromised disease development in a pedigree with a member diagnosed as having X-HIGM. In this case, the R203 site plays critical roles in the interaction with the CD40 receptor on the B-cell surface, which forms at least three hydrogen bonds (Fig. 5). By contrast, the CD40L ${ }^{\mathrm{R} 203 \mathrm{I}}$ variant fails to form hydrogen bond networks with the receptor, which is predicted to deactivate the downstream signaling pathways of B-cell development and antibody class switching (Fig. 6). High-throughput sequencing technologies can detect genetic variations in humans at a single site or numerous sites and have been widely used to study clinical molecular diagnoses. Whole-exome sequencing can quickly identify disease-causing genes and variant sites, and has clear advantages in the detection of monogenic diseases [24]. This way of disease diagnosis is very helpful for early diagnosis, genetic intervention and suitable therapeutic schemes of the disease in the future and further improve the survival rate of the patients and facilitate the development of genetic counselling and prenatal diagnosis.

\section{Conclusion}

Ocur study identified seven rare nonsynonymous SNVs and three genes with rare nonsynonymous SNVs (CD4OLG, $B T K$, and WAS) associated with immunocompromised disease development. Of the seven genes analyzed in the present study, the p.R203I variant of CD4OLG gene may be involved in the development of X-HIGM in this case. However, functional studies should be performed in the future to confirm this finding.

\section{Abbreviations}


CD40LG, CD40 ligand gene; EAS, East Asian population of the ExAC database; ExAC, Exome Aggregation Consortium; gnomAD, Genome Aggregation Database; SNV, single-nucleotide variant; X-HIGM, X-linked hyper-lgM syndrome.

\section{Declarations}

\section{Ethics approval and consent to participate}

The study was approved by the local institutional ethics committee at the First Affiliated Hospital of Anhui Medical and permission was came from the guardians of the patient.

\section{Consent for publication}

All authors have given their consent for the manuscript to be published.

\section{Availability of data and materials}

The datasets generated and analyzed during the current study are not publicly available due to privacy limitations but are available from the corresponding author on reasonable request.

\section{Competing interests}

The authors report no conficts of interest in this work.

\section{Funding}

This work was supported by a grant from Key Research and development project of Anhui Province funding (grant number 201904a07020042).

\section{Authors'contributions}

DSG, SB and JTG had substantial contributions to proposed research and review. HSH and FLL performed the experiments and data curation. WW had substantial contributions to writing, data curation, formal analysis and investigation. DJL and ZYL gave the critical comments. All authors read and approved the final version of the manuscript.

\section{Acknowledgements}

We are grateful for Key Research and development project of Anhui Province funding and parent of the patient.

\section{References}

1. Kroczek RA, Graf D, Brugnoni D, et al. Defective expression of CD40 ligand on T cells causes "X-linked immunodeficiency with hyper-IgM (HIGM1)". Immunol Rev. 1994;138:39-59.

2. Fuleihan, R.L. Hyper IgM syndrome: the other side of the coin. Curr Opin Pediatr. 2001;13:528-32.

3. Franca TT, Barreiros LA, Al-Ramadi BK, et al. CD40 ligand deficiency: treatment strategies and novel therapeutic perspectives. Expert Rev Clin Immu. 2019;15:529-540.

4. Qamar N, Fuleihan RL. The hyper Ig M syndromes. Clin Rev Allerg Immu. 2014;46:120-130. 
5. Vogel LA, Noelle RJ. CD40 and its crucial role as a member of the TNFR family. Semin Immunol. 1998;10:435442.

6. Notarangelo LD, Hayward AR. X-linked immunodeficiency with hyper-IgM (XHIM). Clin Exp Immunol. 2000;120:399-405.

7. De la Morena MT, Leonard D, Torgerson TR, et al. Long-term outcomes of 176 patients with X-linked hyper-lgM syndrome treated with or without hematopoietic cell transplantation. J Allergy Clin Immun. 2017;139:12821292.

8. Levy J, Espanol-Boren T, Thomas C, et al. Clinical spectrum of X-linked hyper-lgM syndrome. J Pediatr. 1997;131:47-54.

9. Lin SC, Shyur SD, Ma YC, et al. Hyper-IgM1 syndrome with interstitial pneumonia and diarrhea caused by coxsackievirus B4 in a 3-month-old infant. Ann Allerg Asthma Im. 2005;95:93-7.

10. Davies EG, Thrasher AJ. Update on the hyper immunoglobulin M syndromes. Brit J Haematol. 2010;149:167180.

11. Gallagher J, Adams J, Hintermeyer M, et al. X-linked Hyper IgM Syndrome Presenting as Pulmonary Alveolar Proteinosis. J Clin Immunol. 2016;36,564-570.

12. Torabizadeh $M$, Nabavi $M$, Zadkarami $M$, et al. X-linked hyper-lgM syndrome associated with pulmonary manifestations: A very rare case of functional mutation in CD40L gene in Iran. Curr Res Transl Med. 2019;67:2830 .

13. Jesenak M, Banovcin P, Jesenakova B, et al. Pulmonary manifestations of primary immunodeficiency disorders in children. Front Pediatr. 2014;2:77.

14. Barbouche MR, Chen Q, Carbone M, et al. Comprehensive review of autoantibodies in patients with hyper-lgM syndrome. Cellular \& Molecular Immunology. Cell Mol Immunol. 2018;15:610-617.

15. Lleo A, Liao J, Invernizzi P, et al. Immunoglobulin M levels inversely correlate with CD40 ligand promoter methylation in patients with primary biliary cirrhosis. Hepatology. 2012;55:153-160.

16. Mavroudi I, Papadaki HA. The role of CD40/CD40 ligand interactions in bone marrow granulopoiesis. Sci World J. 2011;11:2011-2019.

17. De La Morena MT. Clinical phenotypes of hyper-IgM syndromes. J Allergy Clin Immunol Pract. 2016;4:10231036.

18. Ma YC, Lee WI, Shyur SD, et al. De novo mutation causing X-linked hyper-lgM syndrome: a family study in Taiwan. Asian Pac J Allergy Immunol. 2005;23:53-59.

19. Meng X, Yang B, Suen WC. Prospects for modulating the CD40/CD40L pathway in the therapy of the hyper-IgM syndrome. Innate Immun. 2018;24:4-10.

20. Jain A, Kovacs JA, Nelson DL, et al. Partial immune reconstitution of X-linked hyper Ig M syndrome with recombinant CD40 ligand. Blood. 2011;118:3811-3817.

21. Mazzei GJ, Edgerton MD, Losberger C, et al. Recombinant soluble trimeric CD40 ligand is biologically active. J Biol Chem. 1995;270:7025-7028.

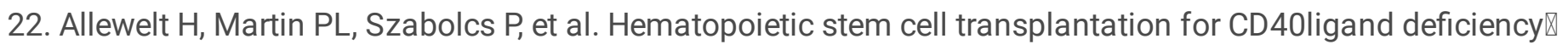
Single institution experience. Pediatr Blood Cancer. 2015;62:2216-2222.

23. Hubbard N, Hagin D, Sommer K, et al. Targeted gene editing restores regulated CD40L function in X-linked hyperIgM syndrome. Blood. 2016;127:2513-2522. 
24. Wang TJ, Wu LF, Chen J, et al. X-linked hyper-lgM syndrome complicated with interstitial pneumonia and liver injury: a new mutation locus in the CD40LG gene. Immunol Res. 2019;67:454-9.

\section{Table}

Table 1 Three pivotal gene variants associated with immunodeficiency-related diseases

\begin{tabular}{|lllllll|}
\hline $\begin{array}{l}\text { Gene } \\
\text { symbol }\end{array}$ & Cytoband & $\begin{array}{l}\text { SNV } \\
\text { annotation }\end{array}$ & $\begin{array}{l}\text { ExAC } \\
\text { ALL }\end{array}$ & $\begin{array}{l}\text { ExAC } \\
\text { EAS }\end{array}$ & $\begin{array}{l}\text { gnomAD } \\
\text { ALL }\end{array}$ & $\begin{array}{l}\text { gnomAD } \\
\text { EAS }\end{array}$ \\
\hline WAS & Xp11.23 & NM_000377:exon11:c.C1378T:p.P460S & 0.0048 & 0.0382 & 0.0023 & 0.0343 \\
BTK & $\mathrm{Xq22.1}$ & NM_001287344:exon18:C.C2001T:p.C667C & 0.3208 & 0.4463 & 0.3666 & 0.4289 \\
\hline CD40LG & & NM_000074:exon5:c.G608T:p.R2031 & ND & ND & ND & ND \\
& Xq26.3 & & & & & \\
& & & & & & \\
\hline
\end{tabular}

Abbreviations: EAS, East Asian population of the ExAC database; ExAC, Exome Aggregation Consortium; gnomAD, Genome Aggregation Database; ND, not determined; SNV, single-nucleotide variant

\section{Figures}

I

II

III
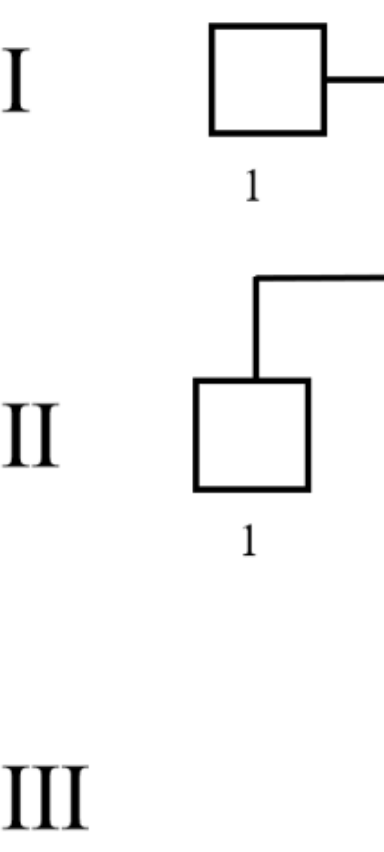$$
\sqrt{2}
$$

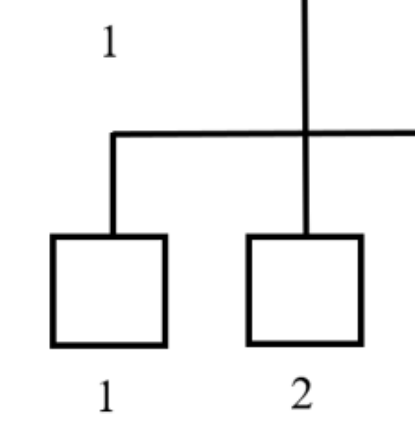



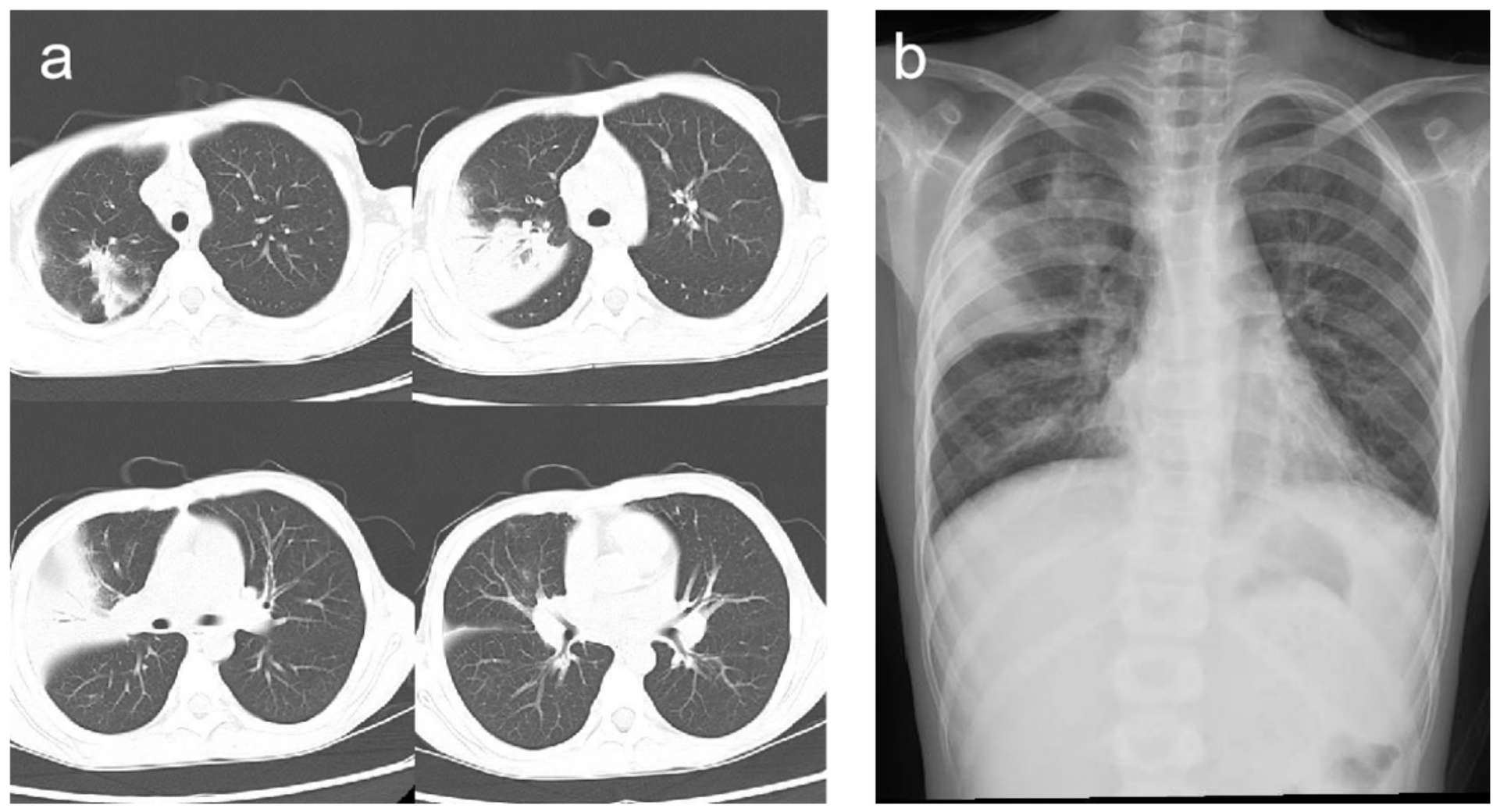

Figure 2

Chest Computed Tomographic Scan (a) and Chest X-ray (b): The imaging results of the child's chest show inflammation of the right upper middle lobe and mild bronchiectasis with infection of the bilateral inferior lobes
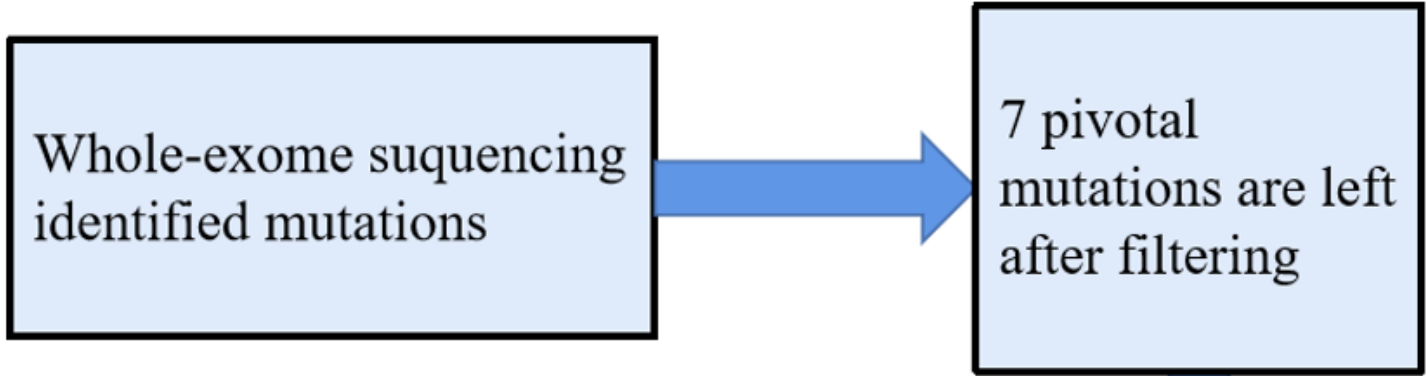

4 genes have not been reported to be involved in X-HIGM

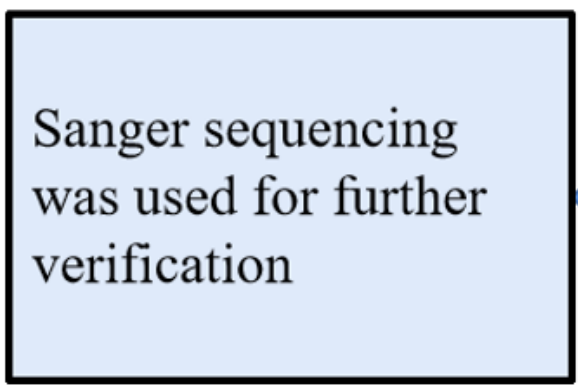

3 genetic variants associated with immune deficiencyrelated diseases were identified 
Figure 3

Flowchart for Variant Screening: Seven pivotal genes with variants were selected from the whole-exome sequencing results. Of them, three genes with nonsynonymous variant sites were identified as potentially involved in immune deficiency-related diseases. Sanger sequencing was used for further verification of the variants

III1

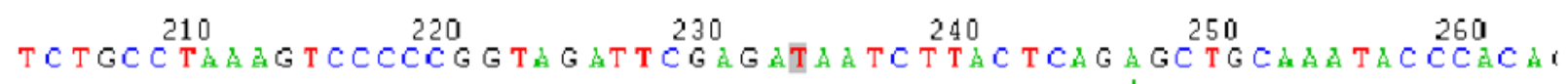

II3
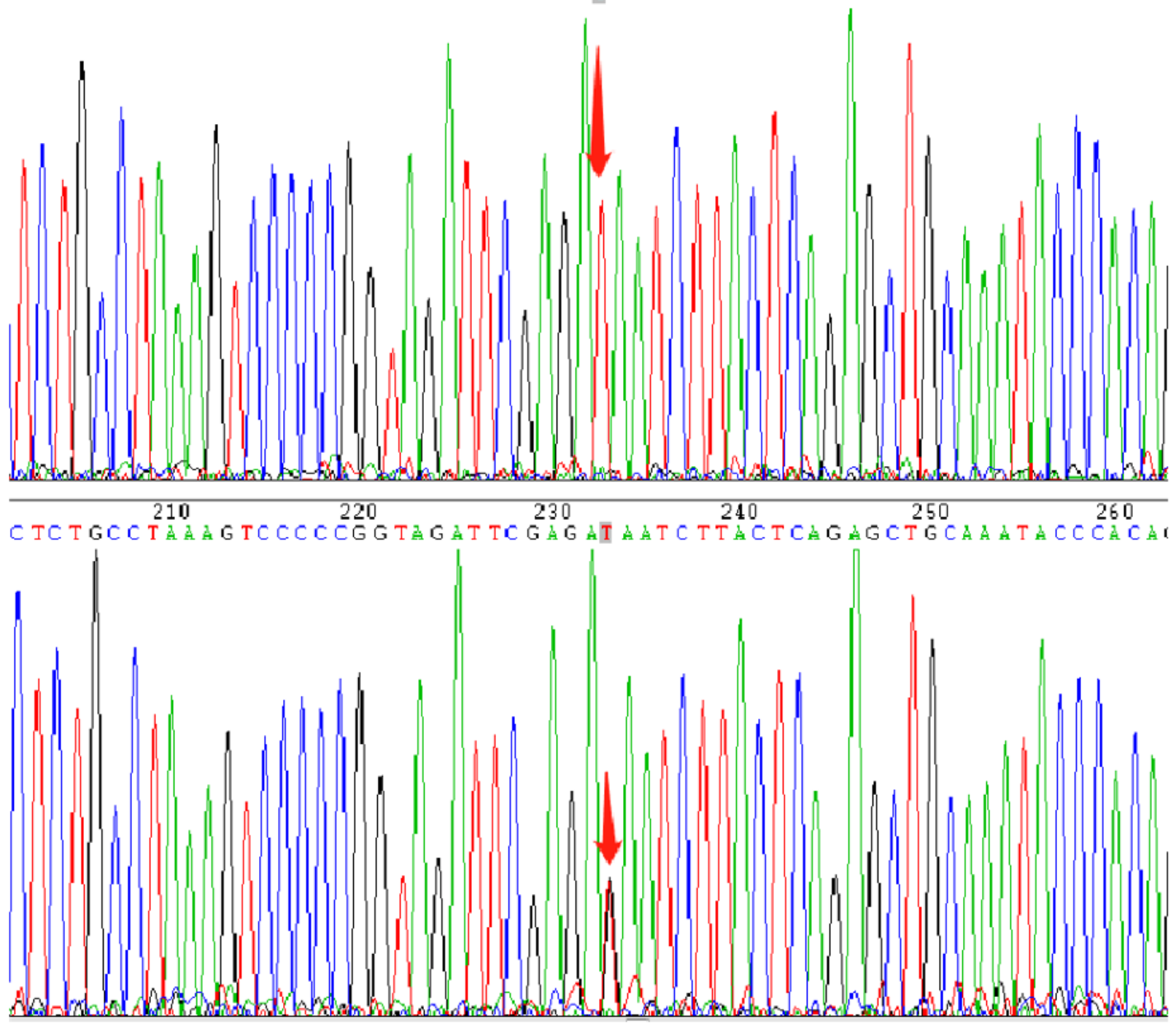

Figure 4

Sequence Electropherogram of CD40LG 


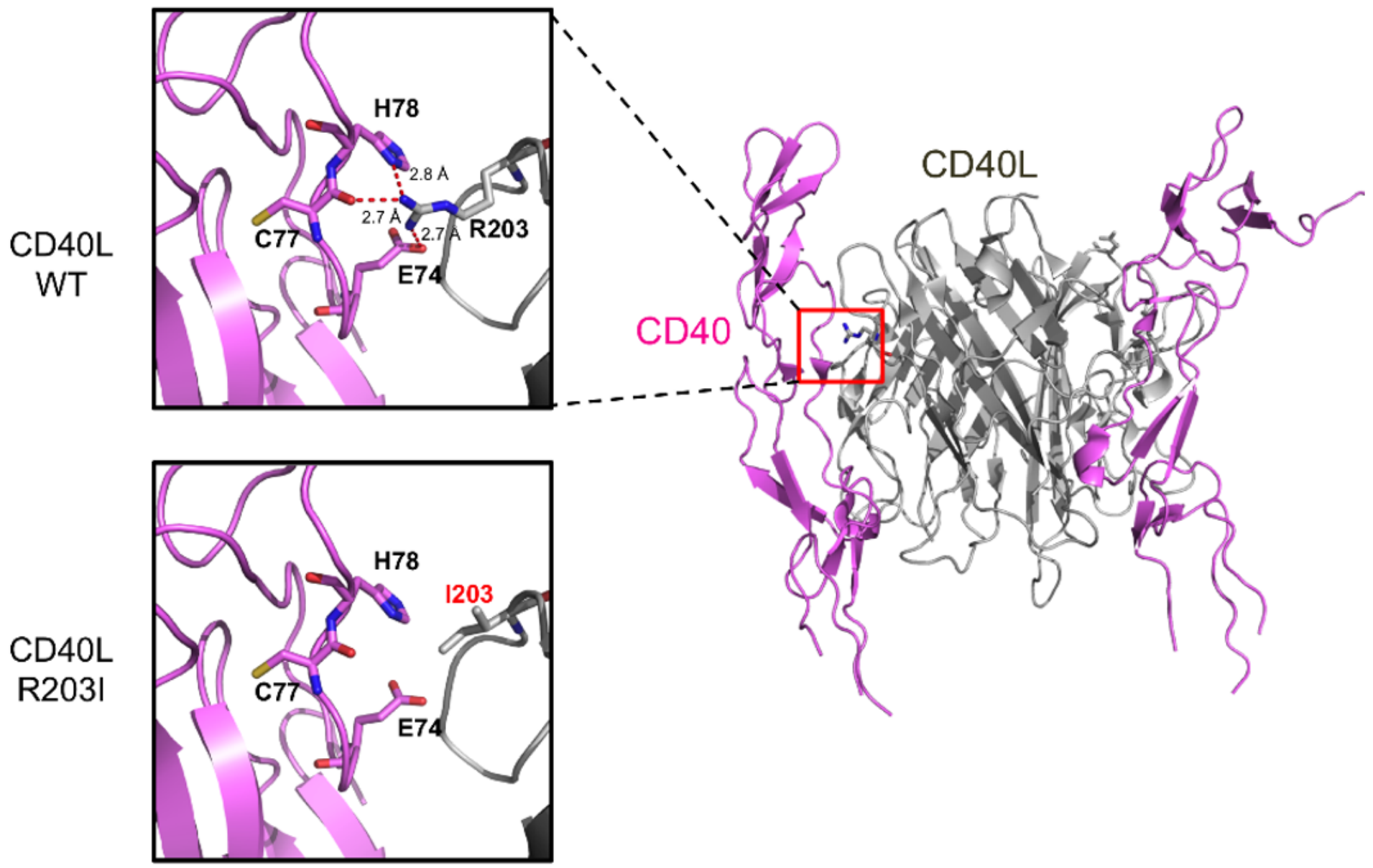

Figure 5

Analysis of Molecular Mechanisms: Structural modeling predicts the CD40LGR203I variant is unable to form hydrogen bond networks with the CD40 receptor, which fails receptor oligomerization on trimeric CD40L. The modeling is based on the CD40:CD40L complex crystal structure (PDB 3QD6) 


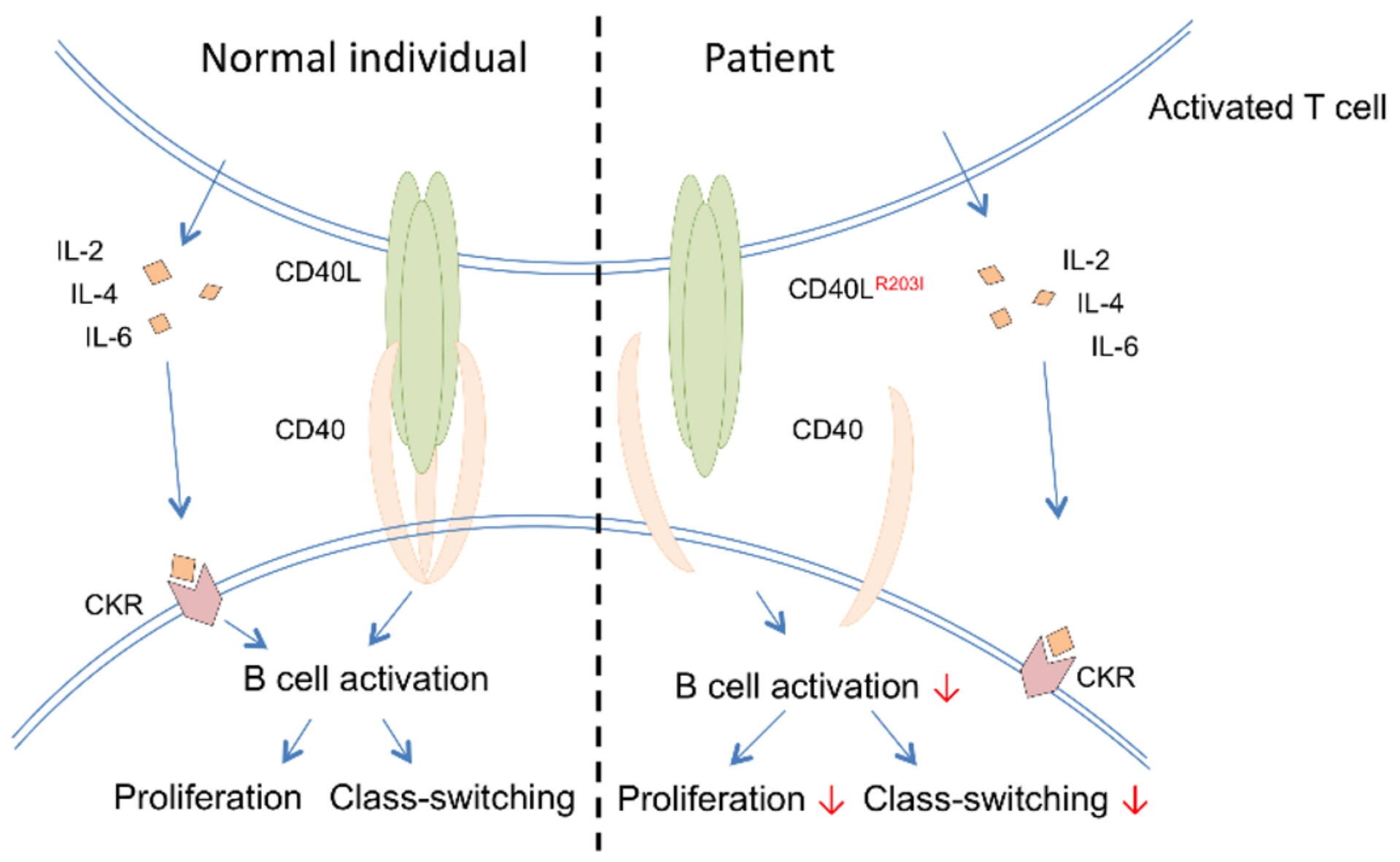

Figure 6

Working Model of CD40LG Variant-Induced X-HIGM in this Case 